\title{
New uses for the on-line computer medium in mental health care
}

\author{
JAMES H. JOHNSON, WILLIAM HARRIS, CHRISTINE THOMPSON, SANDER MARCUS, \\ DEENA BLOCK, KATHY NOVAK, NANCY YINGST, ADRIENNE ALLERT, GLEN HOBIN, \\ ELIZABETH BYRNES, DENNISE STRAUCH, CRAIG FELDMAN, \\ DAWN NIEDNER, and ANDREA FINK \\ Illinois Institute of Technology, Chicago, Illinois 60616
}

\begin{abstract}
A number of research projects related to new uses for computers in mental health service delivery are presented. These projects include work on software psychology, new computer applications in mental health care, and studies relating to the successful implementation of technology in mental health settings. Emphasis is placed on developing a better understanding of appropriate uses of technology rather than merely describing new applications.
\end{abstract}

In a review of the history of the use of computers for mental health treatment services, Johnson, Giannetti, and Williams (1976) suggested that there were two major developmental trends in the area: automated administrative data systems and automated clinical techniques. At that time, most of the work on the use of computers in the mental health sector had focused on mimicking then current clinical and administrative procedures. They further suggested that real advances in the use of on-line computer technology for mental health care delivery would come after a third line of development, interventionally relevant automated systems, was widely evident.

In a related paper, Giannetti, Klingler, Johnson, and Williams (1976) noted that the on-line computer medium is unique among previous media in the mental health field and, therefore, will require a new methodology to extract its true promise. It was their opinion that new assessment and treatment techniques that were developed specifically for on-line computer technology would be required. In these two papers, they were suggesting that the future promise of the use of computers in mental health would not truly be fulfilled if behavioral scientists were to continue to automate procedures that were really more appropriate for previously developed media and were descriptive rather than interventional in their focus. They were calling for creative research that would lead to new ways of delivering services through the on-line computer medium.

Unfortunately, very little work in this new area has been reported since 1976. Most of the newer work on the use of computers in mental health continues to represent a refinement of old methodologies. It is for this reason that we thought it would be interesting to report on developments within our laboratory at the Illinois Institute of Technology (ITI). Current research there spans both old and new approaches to computing in mental health, but these applications also reflect methodologies and techniques that are the most contem- porary in the field. In this paper, we will describe a number of research projects and suggest how these represent new and useful approaches.

\section{COMPUTER HARDWARE AND SOFTWARE}

The basic hardware upon which the present work is built is an LSI 11/03 with 32,000 words of memory, two floppy disks, a VT 100 cathode-ray tube (CRT) terminal, and a Diablo printer. All software is in BASIC running under RT-11.

\section{RESEARCH PROJECTS}

Our research is multifaceted. Some of us are working on ways of improving man-machine interactions, called "software psychology" by computer scientists. Others are working on specific applications in the mental health care area. Still others are working in related areas, such as readiness for implementation of computerized techniques in mental health care delivery systems. Throughout these research projects, we have been intent on developing knowledge that will lead to better acceptance of technology in mental health care.

\section{Software Psychology}

The general scope of software psychology has been delineated by Schneiderman (1980). We have become interested in one aspect of it: the interface between the naive user and input devices and output data. This area is important to us because the acceptance of computers in mental health care delivery hinges, to a large degree, on the ability of patients to input data appropriately and of staff to make use of output data. Without these occurrences, it is likely that even the best new applications in the field will fail to gain acceptance.

On the input side, we have been considering such questions as whether one key entry (with a "back-up" feature for error correction) or a two-key entry system 
(datum followed by a "send") leads to better satisfaction and fewer errors. Other questions pertain to the effects of variable computer response latencies, different types of key arrangements, and changing the "tone" of instructions.

Problems related to output concern the effects of repetitive prose from report to report, type of format that has greatest reader impact, and techniques of retrofitting to combine multiple outputs.

Certainly, we have not yet made many advances in this area, but the recent paper by Johnson and Novak (Note 1) is the first to consider the problem of questionnaire response bias due to gathering test answers with a computer terminal. We expect that continued work in this area will help use to develop computerized applications that are human engineered to the needs of the field and will lead to enhanced acceptability of technology in mental health service delivery.

\section{New Mental Health Applications}

On-line Rorschach interpreter. Although a number of computerized interpretation systems have been developed for nonprojective personality inventories, this has not been the case with the Rorschach. The complexity of administration, data collection, and scoring prevent a fully computerized method's being clinically and economically feasible. Because of this, our work has been limited to the development of an on-line interpretation and report method.

For almost 2 decades, Piotrowski $(1964,1980)$ has been the major researcher attempting to computerize Rorschach interpretations. Other psychologists working in personality assessment have not actively pursued an automated Rorschach approach for several reasons. First, the lack of a unitary scoring system emerges as a major barrier. (The Rorschach has five different scoring systems; see Exner, 1969.) Moreover, many clinicians have developed personalized scoring methods and others have abandoned structural analysis, relying solely on content for interpretations. Second, the reliability and validity of the Rorschach have been questioned and have been found to lack empirical support (Klopfer, 1968). Third, ardent proponents of Rorschach psychology have condemned on-line interpretations. They contend that computer-assisted procedures are meaningless because the automated approach overlooks the dynamic quality of the data. Finally, the status of the Rorschach has declined considerably, causing some to forecast its end as a viable personality assessment tool (Knutson, 1972).

Within recent years, there has been renewed interest in research on the Rorschach (Klopfer \& Taulbee, 1976). Although the reasons for its revitalization can be attributed to numerous factors, the introduction of Exner's (1974) Comprehensive Rorschach System has been a major impetus.

The Exner (1974) system incorporates significant parts of the five major Rorschach systems. The principal mode of interpretation is based on a structural analysis of the data. The system contains norms that are age specific and are grounded in rationales that have empirical support. The structural nature of the raw data and the reliability and validity of the scoring method are major reasons for creating an on-line interpretation that is based on the Exner system.

We have nearly completed development of an on-line computerized Rorschach interpretation procedure. The starting point for the development of this on-line approach was Exner's (1978) work. Exner's clinical teachings were culled to find a series of almost 100 rules relating protocal characteristics and personality. Three interpretive output statements were written for each rule. These statements serve as the explanation for the scores that are obtained from the structural summary. (The reason for including three statements is to provide a more diversified, human engineered, report.) When the data are applicable to a specific rule, an appropriate interpretive statement is randomly selected from three comparable interpretations.

A simple listing of interpretive statements, while useful, does not provide a report reader with a document that synthesizes the information found in the protocol. A more effective report method has been developed that outputs interpretive statements in a paragraph form and covers specific areas of personality functioning. Areas include: intellectual functioning, emotional state, character and attitude, interpersonal relations, primary defense mechanisms, and treatment. All statements are related to specific paragraphs (i.e., an interpretive statement describing an aspect of the person's interpersonal functioning is assigned to a paragraph in this area). We also have developed a method to eliminate contradictive and duplicative statements. Because of these additions, the program results in a document that is more appealing to clinicians.

An on-line objective personality test. The Psychological Systems Questionnaire (PSQ) was initially constructed by Johnson, Giannetti, and Williams (1979) as a result of their experience in developing an on-line computer-assisted psychological assessment system (PAU) at the VA Hospital in Salt Lake City (Johnson \& Williams, 1975). The PAU was designed to collect data for treatment planning. People requesting care are administered an extensive battery of psychological tests on CRT terminals. The responses are collected on a keyboard and transmitted directly for computer processing. The interpretation is then printed in time to assist the clinician in triage decision making. However, the PAU process is extremely lengthy, involving nearly 2,000 questions and taking several hours to complete. In an effort to shorten the testing process while maintaining the depth and range of questioning, work on the PSQ began.

The original development of the PSQ is presented in detail in two recent papers (Johnson \& Giannetti, 1979; Johnson et al., 1979). Items from the PAU data base were rationally categorized. Five senior clinicians 
were asked to examine and group these items. Further revisions were made to the category headings after contacting experts in clinical care and psychopathology throughout the country. The final categorization covered the following areas: demographic data, dissimulation, social history, vocational background, family background, readiness to accept treatment, genetic background, personality, quality of living environment, psychopathological symptoms, and somatic problems.

Approximately 300 items were written for each domain, to form a series of construction tests. These construction tests were administered to $600-900$ normals and psychiatric patients from mental health facilities in the U.S. and Canada and from the general population in the state of Utah. All construction tests were factor analyzed to reduce the item pools and to determine the unique contribution of each item to its respective domain. Finally, a group of rational multiple-choice questions was added to the beginning in order to provide demographic information and rational information for test-item branching.

The resulting PSQ consists of 32 multiple-choice demographic and branching questions, 18 dissimulation questions and 711 true-false questions. All assessees answer the demographic questions and the dissimulation questions. Responses to the demographic questions determine which questions are to be eliminated from the item pool, and responses to the dissimulation questions indicate whether or not to present the remaining relevant questions.

A computer program for the administration, scoring, and interpretation of the PSQ has been developed. It is designed for flexibility and adaptation. As the PSQ continues to be updated, so will the program for administration, scoring, and interpretation.

Questions are presented according to a logical interrogatory branching sequence as opposed to a fixed linear sequence of questions. Through the use of this flexible systems design, a large item pool is reduced and individualized to allow a comprehensive, detailed and efficient assessment of each person who is tested. With certain questions, obvious rational branching systems are used. For example, a person who is an only child is not asked questions pertaining to siblings. The verbal content of each sentence is also sensitive to the subject's individual characteristics. For example, a person who has been divorced or widowed may be asked questions concerning his "ex-wife" or his "deceased wife."

Not all questions lend themselves to a rational branching scheme. For example, questions pertaining to psychotic, depressive, and obsessive/compulsive character disorders do not. In these instances, an adaptive testing technique developed by Wood (1973) is employed. Wood's technique involves fixed strategies for question presentation. In the case of the PSQ, rules are specified that apply to all subjects. All subjects are asked a small sample of questions from the psychosis scale, depression scale, obsessive/compulsive scale, and so on. The ques- tions chosen for initial presentation are designed to measure minimal indications of these disorders. The computer checks the assessee's responses on these items against predefined criteria and then makes a decision as to whether the remaining questions in the scale should be presented. These minimal indicator questions were first selected through the judgment of several experienced psychologists and later subjected to a part-whole correlational analysis to verify item homogeneity.

To guard against inappropriate responses that would invalidate the rational data collection, a validity scale is administered prior to all other questions. This validity scale was adapted from the dissimulation index (the Q1) developed by Johnson, Williams, Klingler, and Giannetti (1977). It is a brief set of 18 questions that check for the accuracy and validity with which a person responds to computer-presented items. Subjects are administered the entire PSQ item pool only if they obtain a passing score on this scale.

The methods employed for report productions are based upon the PROSE program developed by Johnson, Cole, and Williams (1975). However, the reporting techniques for the PSQ have been expanded to incorporate recent advances in the area of computerized report writing (Johnson et al., 1977). These advances include the use of random sentence stems and retrofit programming.

In examining the interpretative material from the PSQ item pool, words, phrases, or sentences relating to numerous areas of patient functioning are apparent. In order to promote readability of the report, the diverse PSQ topic areas have been integrated and arranged in the manner generally adopted by clinicians. The topic areas include the following: validity, current distress, prominent symptoms, social history, feelings, attitudes and character, interpersonal relationships, vocational and marital adjustment, diagnosis, and prognosis. All appropriate output statements from the PSQ program are organized according to these various topic areas. They, then, are modified so that the relevant content categories can be presented to the test taker in a tentative way immediately following PSQ administration, scoring, and interpretation. For example, statements suggesting that the patient is "self-critical," "indecisive," "tense," and so on, are presented to the assessee in the following manner via the CRT:

Results on this test suggest that you are
(a) self-critical
(b) indecisive
(c) tense/nervous

The assessee is then asked to rank these problems in order of importance to him/her. He/she is also asked to indicate which statements are incorrect. For those marked incorrect, the computer asks for further information in the form of

Have you suffered from this problem:

(a) only rarely, or in special situations? 
(b) in the past but not now?

(c) only to a very minor degree?

(d) not at all?

As a follow-up to the assessee's rank ordering of problems, questions concerning the circumstances and severity level of the problems ranked as most important will be asked. For example,

You ranked tension as an important problem. Which of the following are most true about that?

(a) It makes it difficult to interact socially.

(b) It is with me all the time.

(c) It makes it difficult for me to live an ordinary life.

(d) It comes and goes, but it is not serious when it comes.

(e) It comes and goes, but it is serious when it comes.

(f) It comes only at specific times.

The severity level may be probed for by asking,

How would you describe your tension?

(1) severe

(2) moderate

(3) mild

This procedure is repeated for all the relevant problem areas specified by the assessee. At the conclusion of this probing, the subject is asked if he/she wants to type any comments about the results that were presented to $\mathrm{him} / \mathrm{her}$. The final report includes the problem areas predicted from the test findings and is organized or retrofit to the comments made by the test taker.

By adopting innovative retrofitting techniques, the final PSQ report is individualized. However, the report might still appear rigid and stilted if the computer program were to employ previous "skeleton narrative" approaches such as those outlined in the PROSE program (Johnson et al., 1975). Thus, a random sentence stem design is utilized in conjunction with the retrofit strategy. The random sentence design utilizes several sentence fragments for each descriptive statement. For example, rather than using solely the stem, "Suicidal tendencies prevail in this subject," the program has alternative sentence stems from which to draw upon: for example, "Test results suggest that this subject manifests suicidal tendencies," "Suicidal tendencies should be recognized in this subject," or "This subject has prominent suicidal tendencies." These alternative sentences are presented randomly so as to vary the wording of consecutive PSQ reports. Finally, the sentences are coded to insure that the narrative flows smoothly from one sentence to the next. The combined use of retrofit programming innovations and random sentence stem design serves to enrich the computerized PSQ reports and, thus, to expand and maximize its clinical value and service.

At this time, all interpretations are rational, drawing on the skill of experienced clinicians. The use of the Q1 screening device helps insure a valid data base from which to build rational interpretations. In the future, as on-line PSQ data are collected, our interpretative statements will be modified and updated.
The collegian game. We have developed a computer simulation "college" game for students, to help them learn positive planning methods for coping with the challenges and problems incurred during their college experience. It provides information regarding what situations are likely to arise during college and feedback concerning the impact of the decisions the student makes in these situations.

Dismissed college students tend to manifest more pathology, to be more impulsive, to lack emotional commitment to education, and to seem unable to profit from past experiences (Vaughn, 1968). A college simulation game, therefore, may not help this group. On the other hand, Vaughn found that students who voluntarily withdraw from college do not exhibit these characteristics more than successful students do. An available computer simulation experience may help this group.

The computer can model various real-world processes (Zuckerman \& Horn, 1973). In the college simulation game, the outcome is behaviorally determined. Chance plays little part in determining outcome, although some random events influence the play and call for decision making. Students, having given some personal profile data (with some data preallocated by the game), can learn to budget time and energy in balancing the social, academic, and personal aspects of college life and can be given feedback concerning these social, academic, and personal decisions. The participants engaging in a simulation game emerge with better comprehension of the variables affecting their behavior within the context of the actual complex system of college as it is simulated.

The objective of our computer game is to familiarize students with some of the decisions and problems that need to be handled wisely in order to do well in college. It has been noted (Demos, 1968) that failure to complete college is related to low scholastic achievement, poor motivation, financial problems, and emotional difficulties. Seldom is it an immediate emergency or any current urgent problem; more often, these develop over several weeks or a semester. The college simulation game, therefore, provides an ongoing and accumulative series of situations that the player is challenged to face, and it gives feedback information at the end of each "semester" that shows why the participant has done well or has failed to maintain the level of competence required to eventually win the game (i.e., graduate from college).

Players are introduced to the game via a short description of the procedures and rules. Next, they answer a series of questions in five areas regarding their plans and priorities for the semester: academics, social life, financial arrangements, extracurricular activities, and family responsibilities. These questions require ratings of how important each area is to the player and how much time per week he/she expects to expand in each area.

Next, requirements for registration and graduation are outlined and the student is shown a "catalog" of 
course listings. Some courses have descriptions and the player is given coded information to indicate whether the course is easy or hard and interesting or boring. Each course earns $3 \mathrm{~h}$ credit; $60 \mathrm{~h}$ of credit are required for graduation. Students may register for three to seven courses per semester. An estimate of study time required is provided, depending on whether the courses are hard/ easy and interesting/boring. Courses that do not have this rating available to the player have nonetheless been covertly rated; the student must depend upon luck when registering for these particular courses.

An example of a course description is the following:

Algebra I. Mitchell Smurtz

Taught by a graduate teaching assistant nicknamed

"Vicious Mitch." Likes to make undergraduates feel

small by giving them huge, tedious assignments. Thinks

teaching is beneath him. Expects to win Nobel prize by

age 25-we predict Booby prize. H:B (Hard:Boring)

Next, players are provided a list of extracurricular activities offered by the college, along with estimates of time requirements. Answers to these questions determine whether a midsemester "crash" will be programmed due to the player's trying to do too much.

After players have registered and signed up for extracurricular activities, they are presented 10 academic situational dilemmas, each of which has three potential solutions. The player may choose to maximize academics, sacrifice academics, or select a compromise solution. Each answer carries predetermined points or penalties. The 10 questions selected each semester are chosen randomly from a longer set of questions established to provide similar time sequences each semester and equal overall point potential. An example of a situational dilemma is as follows:

It's Wednesday night; you're behind in your reading for your hardest class and there's a quiz tomorrow. Your best friend calls and asks you to go out for pizza and beer. You decide to:

(a) Go out for one beer and be back by 10:00 p.m.

(b) Study tonight and tell your friend you'll go out another time.

(c) It's too late to catch up on the reading-you'll go out and enjoy yourself.

Interspersed at random throughout the conflict situations are humorous comments, advice, and opportunities for bonus points.

At the end of the semester, the player receives an evaluation in the five areas previously mentioned. This evaluation is based on several factors. First, all of the information is compared with a rationally derived profile of a good student. Based on this comparison, weights of $0,1 / 2$, or 1 are assigned to the five categories. Weights of $1 / 2$ and 1 indicate an overextension in an area (e.g., trying to work too many hours to earn money). Next, the following formula is applied to the student's scores: $[n(m+1) / 2] / 5$, where $n=$ number of categories assigned $1 / 2$ or 1 and $m=$ summation of scores. The resulting score is added only to those categories (excluding academics) that had weights of $1 / 2$ or 1 . The score for academics (i.e., grade-point average for the semester) is based on an average motivational score (value reported for academic achievement), study time allotted, courses selected, answers on the situational dilemmas, penalties for overextension, and bonus points. The scoring system for each of these areas was selected on a rational basis after a review of the relevant literature.

In addition to an overall evaluation of the semester, the student may receive advice at this time that will help him/her to plan the next semester more wisely. If he/she needs this advice and does not accept it, a penalty is invoked for the following semester; if he/she accepts it, he/she receives a bonus.

An example of a postsemester evaluation is as follows:

\author{
Semester I GPA $=1.9 / 4.0$ \\ Academics: Motivation $=$ High \\ \# of classes: Seven (maximum load) \\ Social Life: Low \\ Financial Arrangements: Full-time job \\ Extracurricular Activities: None \\ Family Responsibilities: Low
}

You studied hard but your full-time job didn't allow you enough time for your studies. Don't despair! You are a diligent worker and if you take the following advice your grades will improve next semester:

(a) Reconsider applying for the National Direct Student Loan for which you are eligible. Visit the Financial Aid Office on campus.

(b) Cut your work schedule to 15-20 hrs. per week.

(c) Sign up for four classes next semester.

(d) Look over the list of extracurricular activities again. With a lighter work load you 11 have time for something besides "hitting the books."

The initial selection procedure is repeated for the following semesters in much the same way as in the first semester. Thus each semester, the student has the opportunity to change his/her basic strategy.

At the end of the final semester, the student will either graduate or not graduate. In the case of the latter, he/she will be encouraged to try the game again and will receive an assessment of his/her performance. Recommendations are also offered, including such advice as taking a study skills course or obtaining assertion training. Bibliotherapy recommendations are optional.

On-line relaxation training. From the time of Jacobson's (1962) classic text on relaxation to Benson and Klipper's (1976) recent literature review, numerous studies have demonstrated the efficacy of relaxation training in reducing stress. We have developed a modification of this proven technology to provide an ordered process of stress reduction on a computerized system.

A key consideration in the present research was the choice of a relaxation training approach that is consistent with the on-line computer medium. The approach that best seems to fit the on-line medium is autogenic training (AT), a system that uses passive concentration in a series of self-controlled and self-monitored discrete steps leading to a physical and mental condition that 
directly opposes stress (e.g., Benson \& Klipper, 1976; Jencks, 1979).

In the standard exercises for AT (Jencks, 1979), the subject sits or reclines in a comfortable position passively concentrates, and repeats brief self-suggestions such as "I am completely quiet and relaxed" or "I am at peace." This is done in brief sessions that are practiced throughout the day. After initial training on preliminary exercises, the subject is gradually introduced to instructions that focus on six steps: (1) a feeling of heaviness, particularly in the limbs, (2) a feeling of warmth in the limbs, (3) concentration on a calm and regular or strong heart beat, (4) focus on calm respiration, (5) a general feeling of comfortable warmth emanating from the solar plexus, and (6) a sensation of coolness in the forehead.

We decided to carefully limit the focus of instructions since the computer method requires no trained person as a monitor. None of the instructions implies anything other than simple relaxation phenomena.

Another consideration in this research is the use of written instructions in place of prerecorded spoken instructions. Although it may seem that spoken instructions are preferable, it must be remembered that each exercise involves the subject's repetition of instructions to himself or herself. A written instruction allows sub. jects to verbalize in their own way, rather than having the instructions contaminated by the auditory characteristics of another spoken voice.

Compacting of an AT series into a single session (as seemed appropriate for a computerized approach) limits the depth and overall effectiveness of the relaxation training, but this can partially be rectified at the conclusion of a session by providing the subject a printout containing recommended instructions for further practice based on his or her performance during the session.

The major strength of compacting is that it provides independent steps that can be continually evaluated and altered as needed. The subject reads the instructions for a particular step, closes his or her eyes on an auditory signal, follows the instructions for that step, opens his or her eyes on a second timed auditory signal, responds at the keyboard to a between-step measure of relaxation, and is ready for the next step. Depending upon that response, the computer can then present an "equivalent" step or a more or less advanced step or terminate the process.

On-line referral center. The on-line medium offers the possibility of providing a means for carrying out a new type of mental health prevention program. At present, many people are difficult to reach or engage in any form of treatment. The on-line computer can be a useful and practical tool for reaching these people. It is very visible, especially if placed in a commonly frequented area. Furthermore, by placing it under the control of the user, fears of being manipulated by a mental health professional can be overcome.
We consider the computer to be a very appropriate modality for use with the population of students at IIT. This population consists of students who most frequently major in engineering. These students tend to be familiar and comfortable with computers and often are rather skeptical of the mental health profession. Nevertheless, their college years can be quite stressful, as they are often separating from families and friends for the first time and considering and planning for a career.

To develop the system, we reviewed 104 Mooney Problem Checklists of clients seen at the IIT Counseling Center. The four problem areas that were reported significantly more often than any of the others were: personal/ psycholgoical relations, adjustment to college work, social/recreational concerns, and social/psychological relations.

Soon we plan to place an on-line computer terminal in the IIT student union. The four most frequently reported areas will be presented to the student, who will voluntarily seat himself or herself at the terminal. The student will then receive a rank-ordered report of the areas of personal concern. The focusing and organizing processes in conjunction with the immediate feedback in the form of a rank-ordered problem list will hopefully lead to heightened self-awareness and decision making ability. Each student's individual score for each area will then be compared with cutoff scores determined by our counseling center data.

In addition to the rank-ordered report, we will make a recommendation or referral on the basis of how the student's scores compare with our counseling center data. The suggested options will be bibliotherapy, referral to the IIT Counseling Center, or referral to a community mental health clinic. Students who fall within a low range of items checked will receive recommendations for readings only. Students scoring in the range that counseling center clients score will receive the suggestion to consider becoming a client at the center (in addition to the readings). Clients scoring in the upper or extreme range will be referred to a community mental health clinic.

\section{Readiness for Use of Computers}

Finally, some of us are working with community mental health centers that either have or are considering the installation of an automated management information system (MIS) (Sidowski, Johnson, \& Williams, 1980). We are concerned with practical questions, such as the optimal organizational climate for such an installation, appropriate and nonappropriate methods of implementing such systems, and the development of measures to determine whether such systems actually have a positive effect on the operation of a mental health center. At present, there is little empirical knowledge, only clinical lore, about these areas. As a result of our research, we hope to learn more about such problems. 
Issues related to organizational climate are being studied through the development of an instrument to categorize the administrative and clinical realities and beliefs of a setting. Beginning with theoretical item pools from the organizational development area, we are putting together a questionnaire that can be administered to mental health center staff members. After a sufficient sample is collected, items will be factor analyzed in an effort to develop an organizational profile of readiness to implement an MIS.

Our approach to understanding the implementation process is much the same. Items are being constructed for administration to various individuals who have been or are involved in the installation of an MIS. Initial results will be factor analyzed to obtain a profile of implementation approaches.

Once good measures for determining the success or failure of an operating MIS are developed, we will administer our organizational setting questionnaire and implementation style questionnaire to personnel in a sample of mental health centers in which MISs have been successful and in a sample in which they have been unsuccessful. We will then attempt to develop a regression equation of the factors in the organizational setting and the implementation process that are related to success. We believe that the results of this process will provide information that will lead to a better understanding of how to go about the proper installation of an MIS.

\section{DISCUSSION}

Our research on the use of computers in mental health ranges from the traditional to the radical, but there is a common thread that runs through all of it. Each project emphasizes the development of knowledge, techniques, and methodologies that will lead to better acceptance of the medium by the field. It may be some years before these innovations are integrated into all technological applications of mental health care delivery. However, we have little doubt about the outcome.

To many, it will seem startling to assert that any new techniques will have a major impact on the use of computers in mental health. However, when one thinks logically about the problems being faced in mental health care delivery, the current approaches available, the previous efforts to computerize these approaches, and the little success that has been obtained by these efforts, perhaps he or she will be similarly persuaded.

For 2 decades now, those of us working on research related to the use of technology in mental health care delivery have been searching for vehicles through which the medium of on-line computerization can be put to its most appropriate use (see Sidowski et al., 1980). It seems to us that some of the approaches described here represent examples of what we have been looking for.

One final aspect of this research deserves comment.
It is being carried out by students and faculty of the clinical psychology program at IIT. Of course, not all of the program members are involved, but it is a sign of the change that is going on in the clinical services sector. Research of this type is now being undertaken by clinicians rather than by experimentalists. It is our belief that as more clinicians engage in this type of research, more techniques will be developed that will enhance progress in the field.

\section{REFERENCE NOTE}

1. Johnson, J. H., \& Novak, K. Human engineering: A computerized testing station. Paper presented at the 22nd Annual Conference of the Military Testing Association, Toronto, 1980.

\section{RETFERENCES}

Benson, H., \& KLipper, M. The relaxation response. New York: Avon Books, 1976.

Demos, J. Analysis of college dropouts-Some manifest and covert reasons. Personnel and Guidance Journal, 1968, 46, 681-684.

EXnER, J. E., JR. The Rorschach systems. New York: Grune \& Stratton, 1969.

EXNER, J. E., JR. The Rorschach: A comprehensive system (Vol. 1). New York: Wiley, 1974.

EXner, J. E., JR. The Rorschach: A comprehensive system (Vol. 2). New York: Wiley, 1978.

Giannetti, R. A., Klingler, D. E., Johnson, J. H., \& Wrlliams, T. A. The potential for dynamic assessment systems using on-line computer technology. Behavior Research Methods \& Instrumentation, 1976, 8, 101-103.

JacoBson, E. You must relax. New York: McGraw-Hill, 1962.

JENCKs, B. Exercise manual for J. H. Schultz's standard outogenic training. Salt Lake City, Utah: American Society of Clinical Hypnosis, 1979.

Johnson, J. H., Cole, E. B., \& Williams, T. A. PROSE: A simple user-oriented program for computer constructed narratives. Behavior Research Methods \& Instrumentation, 1975, 7, 309-310.

Johnson, J. H., \& Giannetti, R. A. Psychological Systems Questionnaire: A decision-relevant patient screening test designed for on-line administration. Journal of Medical Systems, 1979, 3, 75-80.

Johnson, J. H., Giannetti, R. A., \& Williams, T. A. Computers in mental health care delivery: A review of the evolution toward interventionally relevant on-line processing. Behavior Research Methods \& Instrumentation, 1976, 2, 257-260.

Johnson, J. H., Giannetti, R. A., \& Williams, T. A. Psychological Systems Questionnaire: An objective personality test designed for on-line computer presentation, scoring, and interpretation. Behavior Research Methods \& Instrumentation, 1979, 2, 257.260.

Johnson, J. H., \& Williams, T. A. The use of on-line computer technology in a mental health admitting system. American Psychologist, 1975, 30, 388-390.

Johnson, J. H., Williams, T. A., Klinglen, D. E., \& Giannetti, R. A. Interventional relevance and retrofit programming: Concepts for the improvement of clinician acceptance of computer-generated assessment reports. Behovior Research Methods \& Instrumentation, 1977, 9, 123-132.

Kuoprer, W. G. Current status of the Rorschach. In P. McReynolds (Ed.), Advances in psychological assessment (Vol. 1). Palo Alto, Calif: Science and Behavior Books, Inc., 1968. 
Klopfer, W. G., \& Taulbee, E. S. Projective tests. In M. R. Rosenzweig \& L. M. Porter (Eds.), Annual review of psychology (Vol. 27). Palo Alto, Calif: Annual Review, Inc., 1976.

Knutson, J. F. Rorschach. In O. K. Buros (Ed.), Seventh mental measurement yearbook (Vol. 1). Highland Park, N.J: Gryphon, 1972.

Protrowsk1, Z. A. Digital-computer interpretation of ink-blot test data. Psychiatric Quarterly, , 1964, 38, 1-26.

Piotrowsk I, Z. A. CPR: The psychological $x$-ray in mental disorders. In J. B. Sidowski, J. H. Johnson, \& T. A. Williams (Eds.), Technology in mental health care delivery systems. Norwood, N.J: Ablex, 1980.
Schneiderman, B. Software psychology. Cambridge, Mass: Winthrop, 1980.

Sidowski, J. B., Johnson, J. H., \& Willıams, T. A. (Eds.). Technology in mental health care delivery systems. Norwood, N.J: Ablex, 1980.

Vaughn, R. P. College dropouts: Dismissed vs. withdrew. Personnel and Guidance Journal, 1968, 46, 685-689.

Woon, R. Response-contingent testing. Review of Educational Research, 1973, 43, 529-544.

Zuckerman, D. W., \& Horn, R. E. The guide to simulation games for education and training. New York: Research Media, Inc., 1973. 